\title{
Formación académica de formadores en las carreras de Educación de las Universidades públicas y privadas
}

\author{
Grettel Alfaro Rojas ${ }^{1}$ \\ Silvia Alvarado Cordero ${ }^{2}$ \\ Universidad de Costa Rica, Costa Rica \\ Recepción: 29-09-2016 / Aceptación: 01-12-2016
}

\section{Resumen}

Se presentan resultados de la investigación interinstitucional realizada por la Universidad de Costa Rica (UCR) y el Consejo Nacional de Enseñanza Superior Universitaria Privada (CONESUP) del Ministerio de Educación Pública en Costa Rica. El objetivo general fue analizar diversos aspectos relacionados con la preparación del personal docente que imparte los planes de estudio en las carreras de Educación en los grados de bachillerato y licenciatura de las universidades públicas y privadas en Costa Rica.

La metodología utilizada fue de alcance cuantitativo, con información correspondiente a documentos de registro de docentes procedentes de 33 universidades privadas y 4 estatales. Entre las conclusiones se destaca que hay diferencias entre docentes que forman docentes en las universidades públicas y privadas en lo que se refiere a títulos obtenidos, experiencia y publicaciones. Se evidencia que los formadores de formadores de universidades públicas ostentan mayor cantidad de títulos en estudios de posgrado, más experiencia en docencia y en publicaciones. Este estudio brinda aportes importantes para las entidades encargadas de la formación de formadores de la Educación Superior universitaria.

Palabras clave: formación académica, formadores, Universidad pública, Universidad privada.

\begin{abstract}
The results of the interinstitutional research carried out by the University of Costa Rica (UCR) and the National Council of Private Higher Education (NCOPHE) of the Ministry of Public Education in Costa Rica are presented here. The general objective was to analyze the various aspects related to the formation of the teaching staff who develop the curricula of careers in education of high school and undergraduate at public and private universities in Costa Rica.

The methodology used was of quantitative scope, with information corresponding to documents of registry of teachers from 33 private and 4 state universities. Among the conclusions it is highlighted that there are differences between teachers who train teachers in public and private universities in terms of degrees obtained, experience and publications. It is evident that trainers of trainers from public universities hold more diplomas in postgraduate studies, more experience in teaching, and in publications. This study provides important contributions for the entities in charge of the training of trainers of Higher Education institutions.
\end{abstract}

Key Words: academic formation, trainers, public University, private University.

\footnotetext{
1. Asesora Nacional en Curriculum, Consejo Nacional de Enseñanza Superior Universitaria, Ministerio de Educación; email: grettel.alfaro.rojas@mep.go.cr

2. Docente, Universidad de Costa Rica; email: silvia.alvaradocordero@ucer.ac.cr
} 


\section{Introducción}

Uno de los desafíos en investigación de la Educación Superior es enriquecer de manera sustantiva la información disponible en este ámbito, con el objetivo de contribuir en el desempeño de las instituciones y alimentar la planificación estratégica de la educación universitaria.

En este artículo se presentan resultados del proyecto de investigación, fruto de la alianza estratégica entre el Instituto de Investigaciones en Educación de la Universidad de Costa Rica (INIE-UCR) y el Consejo Nacional de Enseñanza Superior Universitaria Privada (CONESUP) ${ }^{3}$, del Ministerio de Educación Pública. Se valora que el trabajo interinstitucional fortalece el área estratégica de la investigación, ya que genera nuevos conocimientos al aplicar metodologías innovadoras para el mejoramiento de la calidad y pertinencia de la gestión de programas, proyectos y actividades académicas de la educación superior universitaria.

El objetivo del estudio fue analizar diversos aspectos relacionados con la preparación del personal docente que imparte los planes de estudio en las carreras del área de educación en los grados de bachillerato y licenciatura de las universidades públicas y privadas en Costa Rica.

Con esta iniciativa de investigación conjunta, se aporta información que contribuye a ofrecer una visión general de las calidades académicas de profesionales encargados de implementar los planes de estudio de las carreras del área de educación.
En el Quinto informe del Estado de la Educación (2015), se reitera la necesidad de contar con mayor información del sector universitario privado, ya que

este faltante de información es un desafío pendiente del sistema educativo que deberá ser resuelto en los próximos años y en el que debe desempeñar un rol protagónico el Poder Ejecutivo, que ostenta las competencias y facultades de regulador de la educación superior privada (p.205).

\section{La Educación Superior en Costa Rica}

La Universidad se visualiza como la entidad social facultada para llevar a cabo el proceso de formación de profesionales, la responsabilidad social es una de las funciones que sustenta su institucionalización. Su labor formal responde a las necesidades de una sociedad y a un contexto específico, que exige dinamismo de adaptación a un contexto en constante transformación.

La Educación en Costa Rica, inició desde 1814, (...) con la creación de la Casa de Enseñanza Santo Tomás, desde este primer impulso, la educación ha tomado un papel fundamental en la historia del país, el cual ha evocado sus esfuerzos en la formación de ciudadanos con altos niveles formativos (Cruz y Sandí, 2014).

En la actualidad, en Costa Rica, la Educación Superior se ofrece en dos ámbitos, a nivel público y a nivel privado, existen un total de 64 instituciones de educación superior uni-

3. Las autoras también se referirán a esta institución mediante sus siglas. 
versitaria ( 5 públicas, 54 privadas y 5 internacionales). La oferta académica en carreras de educación se ofrece en las 5 universidades públicas y en 35 universidades privadas (CONESUP, 2016).

La distribución de oportunidades académicas por ramas del conocimiento para el conjunto de las universidades existentes en Costa Rica, corresponde en su mayoría a carreras de las áreas de Educación, Ciencias Sociales y Ciencias Económicas (58\% entre las tres); y en general, la oferta está más concentrada en las privadas. En estas últimas, dichas áreas del conocimiento absorben el $70.7 \%$ de la oferta, mientras que en las públicas representan el $46.8 \%$ del total de programas. Si se analiza la composición pública/privada en cada área de estudio, se observa que las universidades privadas tienen autorizadas más del $50 \%$ de las carreras en Ciencias Económicas, Ciencias Sociales y Educación (Estado de la Educación, 2015).

Cabe destacar que la población estudiantil que accede a educación superior se ha incrementado de 13000 estudiantes en 1995, a más de 46000 estudiantes en 2013. El aumento en los títulos universitarios otorgados, se podría explicar por "el crecimiento del sector privado de 5.7 veces en éste y 2.1 en el sector público" (Estado de la Educación, 2015, p 206).

Además, según se plantea en el Tercer Estado de la Educación (2011), "en el último quinquenio, en promedio, 10000 nuevos docentes egresaron de las carreras de Educación existentes en el país. De ellos, un 37\% estudió en universidades públicas y el restante $63 \%$ en centros privados" (p. 216). Estas cifras merecen atención de las instancias vinculadas con la Educación Superior.

Cuando se analizan los tipos de grados obtenidos, se denota que la creciente importancia de la educación superior privada ocurre en todos los niveles. En el caso de las licenciaturas, por cada título otorgado en una universidad estatal en el año 2005, se entregaron 2.4 en las privadas; esta relación creció en el año 2013 a 4.2. En bachilleratos y maestrías, el crecimiento fue menor, aunque en ese último año la cantidad de títulos entregados por las universidades privadas duplicaron a los de las estatales.

El aumento de los títulos emitidos por universidades privadas sucedió en un contexto en el que no se crearon nuevas universidades, por lo que se evidencia el aumento del tamaño de las universidades existentes. En el año 2013, las carreras de Ciencias Sociales y Educación continuaron predominando, tanto en los centros estatales (61\%) como en el sector privado (71\%) (Estado de la Educación, 2015).

Tomando en cuenta el crecimiento en el número de instituciones de educación superior y la variada oferta de oportunidades académicas, el Estado costarricense tiene un largo camino por recorrer con respecto a la función de velar por la calidad de la educación universitaria.

Uno de los mecanismos más utilizados en el mundo para elevar y garantizar la calidad de la enseñanza es utilizar estándares internacionales, mediante los sistemas de acreditación 
de instituciones y programas de educación superior, que permiten certificar que una carrera cumple con los requerimientos básicos para poder llamarse de calidad.

En el tema de calidad, se ha planteado como un desafío de la Educación Superior, al existir la necesidad de establecer en el conjunto de Instituciones Públicas y Privadas que la forman y desde las bases jurídicas que norman el quehacer de cada sector, espacios consolidados de interacción, donde la convergencia en torno a altos estándares de calidad de la educación constituya un propósito de acción claro y realizable (IV Informe de Estado de la Educación Costarricense, 2013).

\section{La formación de Profesionales en Educación}

La Educación Superior en Costa Rica se considera “...como principal camino para el desarrollo profesional, en donde las posibilidades a fuentes de empleo y mejores condiciones de vida se incrementan exponencialmente" (Cruz et al, 2014, p.2).

Por lo tanto, es necesario retomar las tareas facultadas a las universidades, para reflexionar acerca de la formación de formadores para la educación y sopesar si la calidad del desempeño del formador es un factor determinante en el sistema educativo.

La sociedad de hoy demanda una educación de calidad que se enfoque en desarrollar, en el aprendiente, las competencias necesarias para utilizar las herramientas y la información a la que tiene acceso, para prevenir y resolver los problemas que se le presentan en su diario vivir, para enfrentarse al cambio y adaptarse al mismo, para generar nuevas condiciones propias y sociales. (Alburez, C.A., León, O., Cifuentes A., M., Achaerandio, L. y Núñez, E., 2013, p.3).

La sociedad debe replantearse el perfil de un formador y exigirle a las universidades su edificación, mediante entes reguladores y supervisores que velen por la calidad del recurso humano educativo. Porque el formador constituye un protagonista más del sistema educativo, se encuentra inmerso en la realidad educativa actual y es también responsable de su evolución.

En materia de formación de profesionales a pesar de los avances, hay un conjunto de temas de gran relevancia para entender el desempeño del sistema educativo. Se requieren estudios que profundicen en la calidad de la formación inicial de los docentes, las modalidades de contratación y el desempeño en las aulas. Asimismo, es preciso investigar y generar información regular y sistemática acerca de la calidad de los ambientes de aprendizaje en los centros educativos, en sus distintas dimensiones: infraestructura, organización, gestión y relaciones sociales (Cuarto Informe Estado de la Educación, 2013).

Como lo menciona Alzate (2015), la formación del profesorado es una acción compleja en sí misma, denota un alto grado de responsabilidad social, le exige a las instituciones implementar currículos novedosos, críticos, abiertos y contextualizados, que posibiliten 
la emergencia de maestros investigadores, reflexivos y transformadores de la realidad.

Por lo tanto, el perfil profesional del docente universitario es un constructo social de cumplimiento ético. Partiendo de este precepto, la universidad con gran responsabilidad selecciona los profesionales óptimos para asumir el compromiso de formar a los peritos o expertos necesarios para una sociedad. De las calidades de los formadores, el dominio y la experticia en su disciplina, las competencias en su desempeño de experto y la actitud que demuestra su compromiso social, dependen la edificación de los profesionales del futuro.

La persona profesional en docencia de docentes tiene el encargo de contribuir con su aporte en la construcción de un profesional desde sus conocimientos, aptitudes, destrezas y las actitudes que le darán el peso ético para un desempeño adecuado. Los profesionales se forman para el mañana, con visión de futuro desde la complejidad de una sociedad en proceso de cambio constante y con la incertidumbre de las transformaciones.

En esta misma línea Alzate (2015, p. 13), subraya que "el mundo de hoy necesita un docente que oriente, facilite, medie e intervenga en los procesos de construcción de conocimiento, de manera que promueva en sus estudiantes la autonomía y las competencias para desempeñarse en aquellos escenarios donde permanentemente interactúa”.
La formación se concibe como un proceso social y cultural que obedece al carácter de la integridad del desarrollo de la capacidad transformadora humana que se da en la dinámica de las relaciones entre los sujetos en la sociedad, en constante y sistemática relación, capaz de potenciar y transformar su comportamiento en el saber, hacer, ser y convivir.

Cabe destacar que con respecto al grado académico de los docentes son el grupo de profesionales con un porcentaje mayor en bachilleres universitarios con un $40.8 \%$, que comparados con otros profesionales solamente el $10.7 \%$ posee un posgrado (Alfaro, Gamboa, Jiménez, Martín, Ramírez y Vargas, 2011, p. 93).

Es necesario que las personas profesionales de la educación, además de su formación con un grado académico, accedan a estudios en el nivel de posgrado: especialidades, maestrías, doctorados o posdoctorados. Esta preparación les brindará herramientas personales y académicas para desempeñarse de manera más eficiente en los contextos educativos.

Los profesionales en Educación de las Universidades tienen la responsabilidad de actualizarse como formadores, para poder alcanzar el momento evolutivo social e histórico de la humanidad y contribuir con la formación profesionales que comprendan la relevancia de su profesión para la sociedad.

La autoformación, se visualiza como un proceso fundamental que posibilita cambios y transformaciones a nivel personal del docente 
y en el contexto donde desempeña su profesión; la formación es un proceso que depende más de las motivaciones intrínsecas, que de lo establecido por las autoridades o entes externos .

Es decir, la formación de profesionales en Educación es una tarea que requiere que se articulen los conocimientos teóricos y la experiencia para lograr propiciar espacios de auténtica trasformación personal y profesional.

\section{Metodología}

La investigación correspondió a un estudio cuantitativo, de tipo descriptivo. Este tipo de estudios busca "especificar las propiedades, las características y los perfiles de personas, grupos, comunidades, procesos, objetos o cualquier otro fenómeno que se someta a un análisis" (Hernández, Fernández y Baptista, 2010, p 80).

Se utilizó la técnica de análisis de contenido, la cual es de gran utilidad pues permite sistematizar y conocer con mayor profundidad un fenómeno objeto de investigación, además de brindarle un carácter científico a la información recopilada y analizada (Tinto, 2013). Con esta técnica se analizaron los documentos de registro de la formación académica de docentes de las universidades públicas y privadas.

La población del estudio corresponde a docentes que laboran en el área de educación en las Instituciones de Educación Superior en el ámbito público y privado, procedente de 33 universidades privadas y 4 universidades estatales.
Para delimitar el marco muestral, se utilizaron los siguientes criterios de inclusión:

- Docentes pertenecientes a universidades que solicitaron actualización de la nómina de docentes entre los años 2007 al 2012.

- Docentes mencionados en las actas del CONESUP, que presentaron la documentación para la actualización de datos.

El marco muestral de las universidades privadas quedó definido por 740 docentes, procedentes de 13 universidades privadas. Para el cálculo del tamaño de la muestra se utilizó el programa STATS ${ }^{\circ}$. Como parámetros de cálculo se consideró un error máximo aceptable de $5 \%$, un nivel de confianza del $95 \%$ y un porcentaje estimado de la muestra del $50 \%$ (el docente tiene una probabilidad de $50 \%$ de ser seleccionado o no). Tomando en consideración estos parámetros, el tamaño mínimo de muestra es de 250 docentes, los cuales fueron seleccionados de manera aleatoria, utilizando el paquete estadístico SPSS versión 19.

Una vez iniciado el proceso de revisión, se encontraron nueve personas repetidas en la lista o bien con información incompleta, por lo que la muestra final quedó en 241 docentes.

También se realizó el contacto con las Decanaturas de las Facultades de Educación de las universidades públicas; se diseñó una base de datos con la información que facilitó cada una de las unidades académicas. El marco muestral resultante fue de 605 docentes. 
En el caso de la Universidad Estatal a Distancia (UNED) y la Universidad de Costa Rica, se decidió seleccionar una muestra por la cantidad de información que se suministró. En el caso del Tecnológico de Costa Rica (TEC) y la Universidad Nacional (UNA), se decidió trabajar con toda la información obtenida. Sin embargo, una vez detallada la información de cada una de las universidades, se excluyeron los casos que contenía información insuficiente. Por lo tanto, se trabajó con la información de un total de 337 docentes de las universidades públicas (UNED, UCR, TEC y UNA).

Se trabajó con la variable formación académica, la cual consideró los indicadores: tipo de universidad donde obtuvo su título, cantidad de títulos, publicaciones realizadas $\mathrm{y}$ experiencia en docencia, según se detalla en la figura 1.

El procedimiento realizado fue diferente, según el ámbito público o privado. Para las universidades privadas, al momento del estudio el
CONESUP, no contaba con una base de datos de docentes, por lo que se procedió a la revisión de las actas de las sesiones realizadas por el Consejo en cinco años (2007 al 2012), para identificar las universidades que realizaban solicitud de actualización de la nómina de docentes. La información se fue ubicando en una base de datos para la elaboración del marco muestral. Una vez seleccionada la muestra, se procedió a la revisión de los 250 informes de docentes, para alimentar la base de datos.

Con respecto a las universidades públicas, la información fue brindada por la dirección de las respectivas unidades académicas de las Facultades de Educación.

Una vez diseñadas las bases de datos se procedió a realizar una limpieza de información, excluyendo los casos incompletos. Los datos obtenidos se sistematizaron en cuadros descriptivos con la información académica de docentes que forman docentes, según tipo de universidad donde estudió, cantidad de títulos, años de experiencia y publicaciones.

\begin{tabular}{|c|c|l|}
\hline Variable & Indicadores & Definición \\
\hline \multirow{4}{*}{$\begin{array}{c}\text { Formación } \\
\text { Académica }\end{array}$} & Tipo de universidad & $\begin{array}{l}\text { Se refiere al sistema de educación superior universita- } \\
\text { rio constituido para funcionar, es decir ámbito público } \\
\text { o privado, del cual obtuvieron el título universitario. }\end{array}$ \\
\cline { 2 - 3 } & Títulos & $\begin{array}{l}\text { Corresponde a la cantidad de títulos académicos obte- } \\
\text { nidos en los niveles de grado o posgrado en Educación. }\end{array}$ \\
\cline { 2 - 3 } & Publicaciones & $\begin{array}{l}\text { Cantidad de producciones académicas realizadas a } \\
\text { nivel nacional o internacional. }\end{array}$ \\
\cline { 2 - 3 } & Experiencia & Cantidad de tiempo dedicado a la docencia universitaria. \\
\hline
\end{tabular}

Figura 1. Definición de la variable e indicadores. 


\section{Resultados}

Con respecto al indicador formación recibida, que corresponde al tipo de universidad privada (a) o pública (b), donde el personal docente que forma docentes obtuvo su título académico; los resultados se presentan en las figuras 2 y 3.

De acuerdo con la información del gráfico, de 241 docentes que laboran en universidades privadas, 189 de ellos reportan título de bachillerato, donde el $80.4 \%$ de profesionales lo obtuvo de universidad privada. Además, 187 profesionales reportan título de licenciatura, siendo el 89.3\% de universidad privada. De 80 docentes que reportan título de maestría, el 97.5\% de ellos es de universidad privada y 3 restantes reportan título de doctorado, representado el 100\% que proviene de universidad privada.

De acuerdo a la información de la figura 3, la mayoría del personal docente ha realizado estudios de grado en universidades públicas. En bachillerato, el 85.2\% de docentes obtuvo el título de una universidad pública. En licenciatura, el $81.5 \%$ de ellos ostenta un título proveniente de una universidad pública. Además, en maestría, el $44.4 \%$ de ellos lo obtuvo de una universidad pública; el 46.7\% estudió su maestría en una universidad privada y el 8.9\% de ellos en universidades en el extranjero. Finalmente, en doctorado, el $27.6 \%$ de ellos proviene de una universidad pública; un $34.5 \%$ de universidades privadas y el $37.9 \%$ de universidades en el extranjero.

En las figuras 4 y 5 se presentan los resultados referidos a la cantidad de títulos obtenidos por docentes que imparten cursos en las carreras de educación de las universidades privadas y públicas.

En el caso de las universidades privadas, el grupo docente posee un promedio de 2 títulos. Se observa en el gráfico que de la población docente que registra título de bachillerato, un $88.9 \%$ reporta tener un título; el $10.6 \%$ reporta ostentar dos títulos y $0.5 \%$ posee tres o más títulos de bachillerato. En licenciatura, un $81.0 \%$ reporta tener un título de esta naturaleza; el 16.9\% reporta dos títulos de esta índole y $2.1 \%$ tres o más títulos de licenciatura. En maestría, un $85 \%$ de los docentes asegura tener un título de este tipo, el 8.8\% dos títulos y $6.3 \%$ tres o más títulos de maestría. Además de la población docente que registra título de doctorado, el 100\% reporta tener un título.

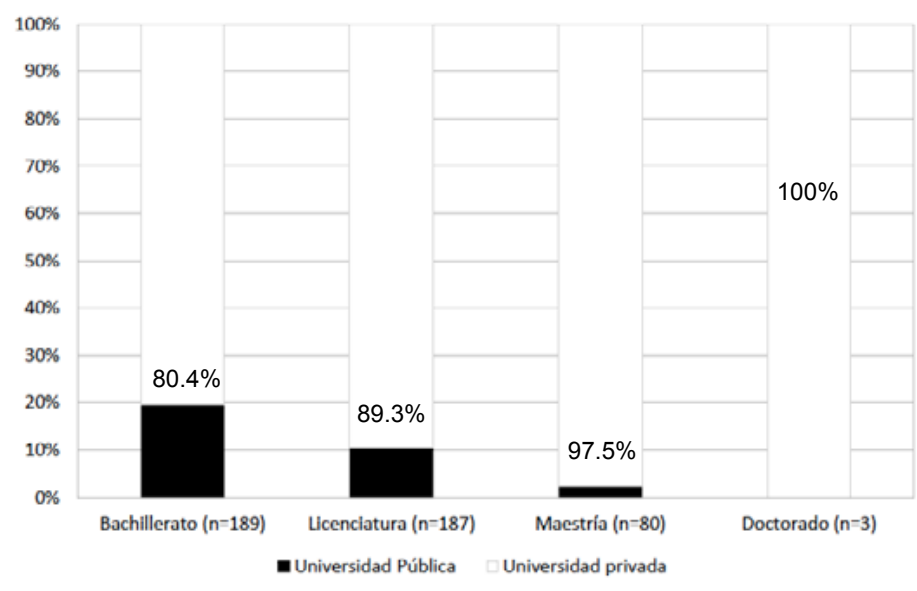

\section{Gráfico sobre clasificación de docentes por Universidad de titulación (a)}

Figura 2. Tipo de universidad donde estudiaron docentes que forman docentes en universidades privadas (total de menciones) $(\mathrm{N}$ : 241), Costa Rica. 


\section{Gráfico sobre clasificación de docentes por Universidad de titulación (b)}



Figura 3. Tipo de universidad donde estudiaron docentes que forman docentes en universidades públicas (total de menciones) (N: 337), Costa Rica.

\section{Gráfico de títulos obtenidos (a)}

Figura 4. Cantidad de títulos en cada grado de docentes que forman docentes en universidades privadas (total de menciones) (N: 241), Costa Rica.

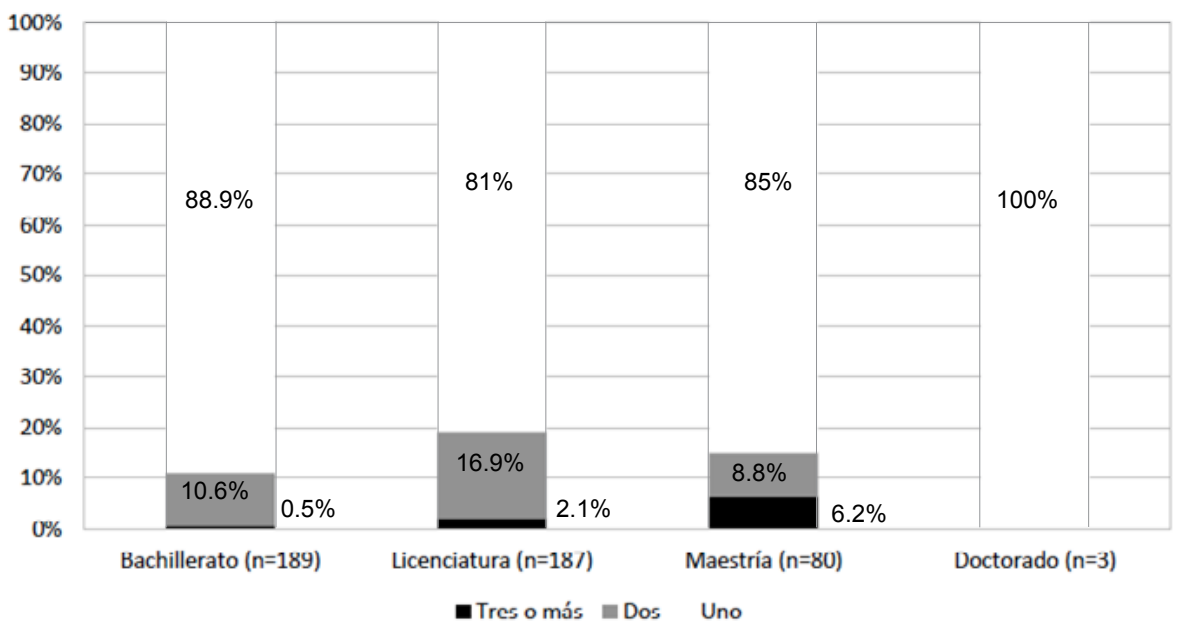

\section{Gráfico de títulos obtenidos (b)}

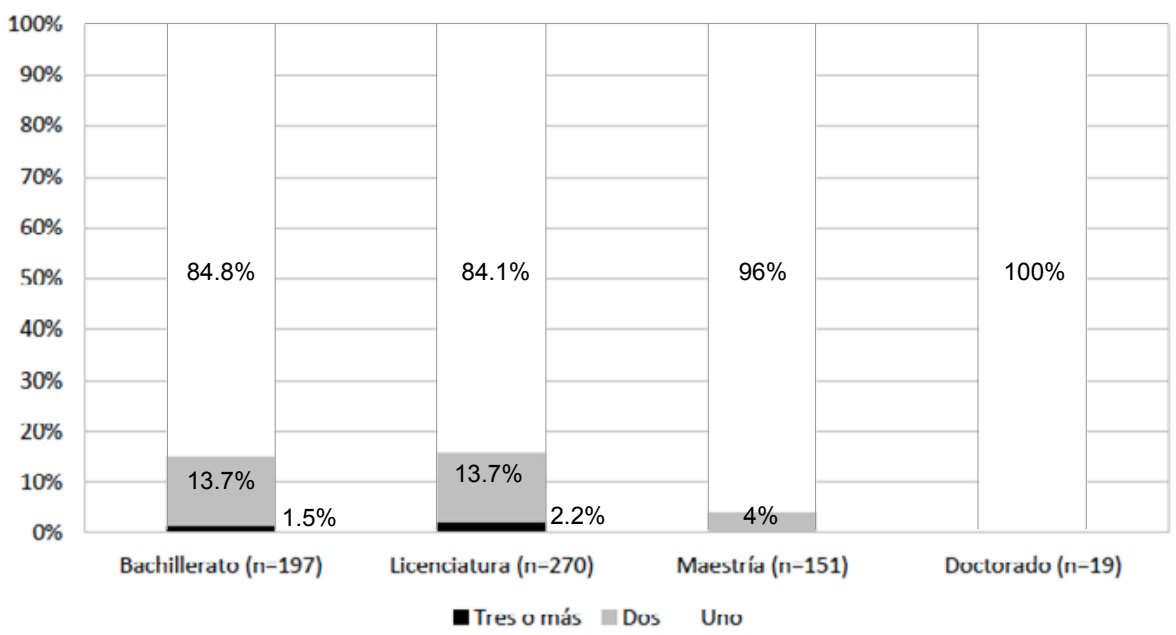

Figura 5. Cantidad de títulos en cada grado de docentes que forman docentes en universidades públicas (total de menciones) ( $\mathrm{N}$ : 337), Costa Rica. 
De los 337 docentes de universidades públicas: en el grado de bachillerato, un $84.8 \%$ reporta tener un título; el $13.7 \%$ ostenta dos títulos de esta índole y $1.5 \%$ tres o más títulos. En licenciatura, un $84.1 \%$ de ellos solo posee un título, el $13.7 \%$ dos títulos y el $2.2 \%$ tres o más títulos de licenciatura. En maestría, un 96\% reporta tener un título y el $4 \%$ manifiesta ostentar dos títulos. Finalmente en doctorado, un $100 \%$ tiene un único título. De este gráfico, cabe rescatar que un $50 \%$ de la población participante registra títulos en los niveles posgrado (maestría y doctorado).

En relación con la cantidad de publicaciones que realizó el profesorado, los resultados de las universidades privadas se detallan en la figura 6 y los de las universidades públicas en la figura 7.

Se evidencia que el $92.9 \%$ de los docentes no posee publicaciones, mientras que $2.8 \%$ manifestó tener de cuatro a quince publicaciones y un $3.7 \%$ de ellos posee de una a tres publicaciones (ver figura 6).

En las universidades públicas, el porcentaje de docentes que realizan publicaciones corresponde a un $75 \%$, los cuales tienen al menos una publicación (7.5\%). El 25\% restante no tiene publicaciones (ver figura 7).

Referente a la experiencia docente con que cuenta el profesorado de las universidades privadas y públicas, este dato se presenta en los gráficos de la figura 8 y 9.

\section{Número de publicaciones por docente (a)}

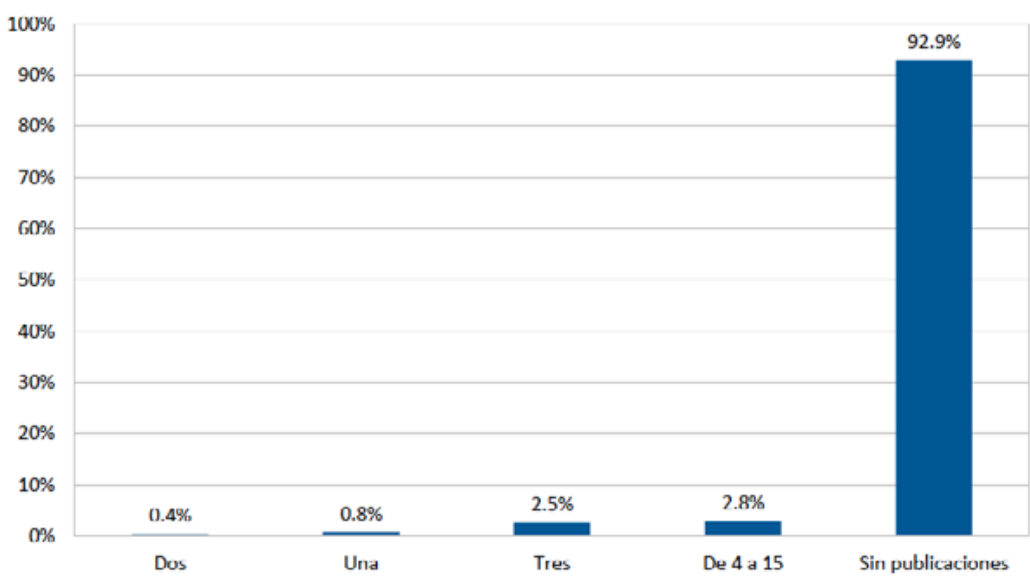

Figura 6. Cantidad de publicaciones de docentes que forman docentes en universidades privadas. (N: 241), Costa Rica.

\section{Número de publicaciones por docente (a)}



Figura 7. Cantidad de publicaciones de docentes que forman Docentes en universidades públicas (N: 337), Costa Rica. 
En los resultados de las universidades privadas, más de la mitad (56.5\%) de docentes que imparten cursos no posee experiencia docente en el momento del estudio.

\section{Número de publicaciones por docente (a)}



- Sin experiencia

- Más de 5 a 10 años

- Mas de un año a 5 años

- Más de 10 afícs a 44 menos

Figura 8. Experiencia de docentes que forman docentes en universidades privadas (N: 241), Costa Rica.

\section{Discusión}

Los resultados del estudio revelan que la gran mayoría de docentes formadores de educadores en universidades privadas han realizado su preparación académica también en las universidades privadas. Por su parte, en las universidades públicas la mayoría del personal docente ha realizado estudios de grado en universidades públicas; sin embargo, para optar por estudios de posgrado consideran tanto instancias públicas, privadas o en el extranjero.

Se considera que la tendencia de las personas de optar por las carreras de Educación, puede obedecer a que es un área en que encuentran múltiples alternativas de estudio, porque carreras del área de Educación se imparten en las 5 universidades públicas y en 35 universidades privadas. El posicionamiento de
De los 337 docentes de universidades públicas, el 59\% de ellos posee experiencia en docencia universitaria de entre 10 a más de 20 años.

\section{Experiencias de los docentes (b)}

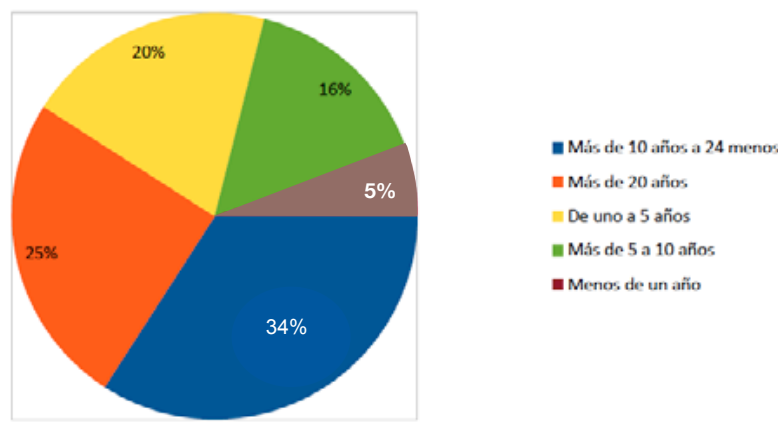

Figura 8. Experiencia de docentes que forman docentes en universidades públicas (N: 337), Costa Rica.

la oferta educativa privada en el país podría responder a varios factores como: duración del plan de estudios, horarios nocturnos y de fines de semana; amplia oferta de universidades que ofrecen la carrera de educación y flexibilidad en apertura de cursos. La legislación de Costa Rica permite que una universidad privada pueda contar con sedes regionales y aulas desconcentradas, facilitándose así que puedan tener presencia en todo el territorio nacional y en el caso de las carreras de educación con una mínima inversión de infraestructura y equipo especializado.

Con respecto a la cantidad de títulos obtenidos en Educación Superior universitaria por los docentes que forman docentes, se encontró que en la actualidad existe una demanda competitiva, que se refleja también en la cantidad de títulos; optando por combinar el tipo 
de universidad pública y privada, especialmente en los niveles de posgrado. Dicha situación, puede obedecer a las posibilidades de acceso, costos, tiempo, especialidad, entre otros.

Los resultados muestran porcentajes muy similares en la cantidad de títulos de bachillerato y licenciatura que tiene un profesional que forma docentes en el ámbito privado o público; solo existe una diferencia en cuanto a cantidad de títulos obtenidos de los niveles de maestría y doctorado, donde los docentes de las universidades públicas registran mayor cantidad.

Esta información podría significar que, dentro del contexto académico se ha convertido en una necesidad que los docentes obtengan varios títulos para mejorar su perfil y carrera profesional. Es importante destacar que para ser contratado como docente universitario, los entes rectores de las universidades públicas y privadas han establecido que se deberá poseer - por lo menos - el nivel académico de licenciatura; y en casos muy calificados y debidamente justificados, se aceptan docentes con nivel académico de Bachillerato Universitario (CONARE, 2013 y CONESUP, 2010).

En Costa Rica, entre los años 2010 y 2011 se emitieron 21446 nuevos diplomas en Educación, que representan más de una cuarta parte del total de títulos emitidos en el país (Estado de la Educación, 2015). De los resultados se infiere que en Costa Rica, las oportunidades de formación en Educación Superior universitaria tienen demanda tanto para el ámbito público como para el privado.
En relación con las publicaciones que realizan el personal académico, se encontró que en las universidades privadas un mínimo de docentes han realizado producción académica (6.6\%), mientras que en las públicas lo han hecho el 75\% de ellos. Se evidencia que en las universidades financiadas por el Estado es alto el porcentaje de docentes que publican, debido a los mecanismos e incentivos con los cuales cuentan para realizarlas.

Para el Consejo Nacional de Rectores (CONARE, 2013), ${ }^{4}$ un docente universitario debe poseer una trayectoria documentada en el campo, mediante publicaciones, experiencia laboral, investigación, proyectos, entre otros. De ahí, la importancia que el personal académico de las universidades logre como parte de su práctica profesional atender estas demandas.

Referente a la experiencia docente, en las universidades privadas llama la atención que más de la mitad de profesionales que imparten cursos no posee experiencia de este tipo. A diferencia de la información brindada por las universidades públicas, que poco más de un tercio del personal académico cuenta con experiencia de entre diez y veinte años en la formación en Educación Superior.

Según la normativa vigente, para la aprobación de una carrera ante el CONESUP, un mínimo del veinte por ciento (20\%) del total del personal académico deberá contar con una experiencia universitaria de al menos de cinco años en docencia, investigación o acción

4. Las autoras también se referirán a esta entidad mediante sus siglas. 
social. Además, al menos un diez por ciento (10\%) del total del personal académico deberá ostentar una experiencia universitaria no menor de diez años.

Estos datos evidencian que la normativa está planteada como recurso para regular la calidad académica del personal docente de las universidades privadas. Sin embargo, el asignar una distribución porcentual para los años de experiencia, favorece que el mayor porcentaje de docentes contratados en las carreras de Educación no la posean. Este criterio podría limitar la calidad académica en las universidades privadas al solicitar que solo un $30 \%$ de la nómina docente de una carrera tenga experiencia de al menos 5 o más años.

En la información obtenida de las universidades públicas, se encontró que un poco más de un tercio del grupo de docentes cuenta con experiencia docente de entre diez y veinte años. La normativa establecida por CONARE para las universidades públicas, plantea que se debe evidenciar la experiencia universitaria o idoneidad para impartir los cursos y actividades académicas asignadas. Por lo tanto, se muestra en la información que los docentes de las universidades públicas cumplen con el requisito de poseer experiencia universitaria.

Con respecto a las calidades académicas del personal docente que labora en las carreras de educación en las universidades privadas, los títulos en su mayoría fueron obtenidos también en universidades privadas; es decir, quienes tienen a su cargo la formación de docentes son los profesionales que recientemen- te egresan de la universidad. Si se relacionan los hallazgos del estudio con respecto a la experiencia en docencia universitaria, se puede inferir que son docentes que se gradúan e inmediatamente se incorporan a impartir cursos, sin contar con la vivencia y aprendizajes que el ejercicio profesional proporciona, lo cual podría repercutir en la calidad de la formación; $y$ también se hace visible en la escasa producción en publicaciones académicas.

El grupo docente de las universidades privadas posee un promedio de 2 títulos, en un rango de 1 y 6 títulos en el grado. No obstante, esta cantidad disminuye en los niveles de posgrado. Por ejemplo, de los 241 docentes participantes en este estudio, solo 3 poseen un nivel de doctorado. Además, en algunos casos, los docentes universitarios en el ámbito privado pareciera que no desarrollan la capacidad de sistematización para mejorar la práctica como mediadores de los procesos de enseñanza y aprendizaje en la Educación Superior. Esto se evidencia en los resultados, ya que un $92.9 \%$ de ellos no han efectuado publicaciones de su quehacer académico.

Por otra parte, con respecto a los resultados de las calidades académicas de docentes de las universidades públicas, se identifica que recibieron su educación de universidades públicas. Llama la atención que la formación a nivel de posgrado fue obtenida de universidades públicas, privadas y del extranjero. También se evidencia que docentes contratados tienen una preparación a nivel de grado académico (bachillerato y licenciatura) de 
universidades públicas. De estos resultados se podría inferir que en Costa Rica, las oportunidades de formación en Educación tienen demanda tanto para las universidades públicas como para las privadas.

Con relación a la cantidad de títulos de docentes que laboran en las universidades públicas, se subraya que ellos poseen un promedio de dos títulos por docente, en un rango de uno y siete títulos. Los porcentajes más altos se ubican en los niveles de licenciatura y maestría. Un dato relevante es que de los 337 participantes, 19 tienen el grado académico de doctorado.

La experiencia de los docentes de las universidades públicas muestra altos porcentajes, con un promedio de 10 a 20 años de experiencia, lo que podría considerarse un aspecto positivo para la calidad de la formación que se brindan en este ámbito académico, con una mayor trayectoria en la preparación en educación; aunado a mayor producción académica, donde más de un $40 \%$ de docentes ha publicado cuatro o más documentos. Esto último denota que se mantiene el interés y las oportunidades de realizar investigación y actualización en los procesos de formación.

Por lo tanto, se concluye que se cumplió el objetivo de conocer las calidades académicas de docentes que laboran en las carreras de Educación en las universidades públicas y privadas. Asimismo, se encontró que hay diferencias entre docentes que forman docentes en las universidades públicas y privadas en lo que se refiere a formación académica, experiencia y publicaciones.

\section{Referencias}

Alburez, C.A, León, O., Cifuentes A., M., Achaerandio, L. y Núñez, E. (2013) Competencias clave en la formación de formadores. Cultura de Guatemala. 34(2), 76-95.

Alfaro, M., Gamboa, A., Jiménez, S., Martín, J., Ramírez, A., y Vargas, M. (2011). Perfil docente: Fundamentos teóricos y Metodológicos. Heredia, Costa Rica: EUNA.

Alzate O., F. (2015). Prácticas y formación docente: un escenario propicio para promover la investigación educativa en Colombia. Actualidades investigativas en Educación. 15(2), 1-17. Doi: http://dx.doi.org/10.15517/aie.v15i2.18962

Costa Rica, Consejo Nacional de Enseñanza Superior Universitaria Privada (2010). Reglamento General del Consejo Nacional de Enseñanza Superior Universitaria Privada.

Costa Rica, Consejo Nacional de Enseñanza Superior Universitaria Privada (2016). Base de Datos SICONESUP. Ministerio de Educación Pública de Costa Rica. 
Costa Rica, Consejo Nacional de Rectores (2013). Normativas de las Universidades Estatales UNED-UCR-UTN-TEC-UNA.

Costa Rica, Constitución Política de Costa Rica. (1949)

Cruz S., S. y Sandí D., J.C. (2014) Importancia de la Educación Superior en el desarrollo profesional para la población estudiantil. III Congreso Internacional de Educación Superior (CIESUP, Costa Rica). Repositorio Universidad Nacional de la Plata.

González, G.Y., y Varela, L. (2010). La Educación Superior Centroamericana en contexto de construcción de espacios comunes del conocimiento. Actualidades investigativas. 10 (Edición Especial), pp. 1-28. Doi: http://dx.doi.org/10.15517/aie.v10i4.10153

Ley 6693. Creación del Consejo Nacional de Enseñanza Superior Universitaria Privada. (1981). Programa Estado de la Nación, en Desarrollo Humano Sostenible (2011). Tercer Informe Estado de la Educación, San José, Costa Rica: Consejo Nacional de Rectores.

Programa Estado de la Nación en Desarrollo Humano Sostenible (2013). Cuarto Informe Estado de la Educación. San José, Costa Rica.

Programa Estado de la Nación en Desarrollo Humano Sostenible (2015). Quinto Informe Estado de la Educación. San José, Costa Rica.

Sistema Nacional de Acreditación de la Educación Superior. (2016). Lista de carreras acreditadas. Recuperado de http://www.sinaes.ac.cr/index.php?option=com_content\&view=article\&id $=13 \&$ Itemid $=115$

Tinto A., J.A. (2013). El análisis de contenido como herramienta de utilidad para la realización de una investigación descriptiva. Un ejemplo de aplicación práctica utilizado para conocer las investigaciones realizadas sobre la imagen de marca de España y el efecto país de origen. Provincia, (29), 135-173. 